\title{
ENERGY HARVESTING IN AN OSTBC BASED AMPLIFY-AND-FORWARD MIMO RELAY SYSTEM
}

\author{
Batu K. Chalise, Yimin D. Zhang and Moeness G. Amin \\ Center for Advanced Communications, Villanova University \\ 800 E Lancaster Avenue, PA 19085, USA \\ Email: \{batu.chalise, yimin.zhang, moeness.amin\}@villanova.edu
}

\begin{abstract}
This paper investigates performance limits of a two-hop multi-antenna amplify-and-forward (AF) relay system in the presence of a multi-antenna energy harvesting receiver. The source and relay nodes of the two-hop AF system employ orthogonal space-time block codes (OSTBC) for data transmission. We derive joint optimal source and relay precoders to achieve different tradeoffs between the energy transfer capability and the information rate, which are characterized by the boundary of the so-called rate-energy (R-E) region. Numerical results demonstrate the effect of different parameters on the boundary of the R-E region.
\end{abstract}

\section{INTRODUCTION}

Harvesting energy from radio signals of a transmitter is a promising technique that has been successfully implemented in various applications, such as passive radio-frequency identification (RFID) systems [1] and body sensor networks with medical implants [2]. Energy harvesting (EH) can be used for prolonging the network operation time in energy-constrained networks, such as sensor networks, which are typically powered by small batteries and have limited life time. In this context, the authors in [3] propose a wireless communication system in which some terminals do not have fixed power supply and thus need to harvest the energy from signals transmitted by other terminals. In particular, [3] considers a three-node wireless multiple-input multiple-output (MIMO) broadcasting system in which the two receiver nodes harvest energy and decode information separately from the signals broadcast by the common transmitter. The transmitter tries to simultaneously maximize the information transfer to the intended receiver and the power transfer to the EH receiver. Note that [3] extends the study of simultaneous information and power transfer of [4], [5] from the single-input single-output (SISO)

This work was supported in part by Defense Advanced Research Projects Agency (DARPA) under contract no. HR0011-10-1-0062. The views, opinions, and/or findings contained in this article/presentation are those of the author/presenter and should not be interpreted as representing the official views or policies, either expressed or implied, of the Defense Advanced Research Projects Agency or the Department of Defense. Approved for public release. Distribution unlimited. link in the co-located receiver (i.e., information and EH receivers are the same) case to the multi-antenna setup with both co-located and separated receivers.

In this paper, different from previous contributions [3], [4], [5], we envisage a wireless communication system in which a multi-antenna EH receiver coexists with a two-hop MIMO relay system where both the source and relay nodes employ orthogonal space-time block codes (OSTBC) [6] and precoders for data transmission. The relay/destination node of the twohop system uses maximum-ratio combining (MRC) technique for detecting/decoding the source signal. In overall, the relay operates in a half-duplex mode using an amplify-and-forward (AF) protocol. The EH receiver harvests energy from the radio signals transmitted by both the source and relay. Information transfer to the destination node and power transfer to the EH receiver are optimally controlled by properly designing the source and relay precoders. In particular, under the total power constraint of the source and relay, we design the optimal source and relay precoders that maximize the rate for the intended receiver while keeping the power transfer to the EH receiver above a certain predefined value. This predefined value is varied to obtain the boundary of the so called rateenergy (R-E) region which illustrates the tradeoffs for maximal information rate versus energy transfer. To the best of our knowledge, simultaneous transfer of energy and information for the proposed system model has not been addressed before, although optimal MIMO relay precoder designs have been solved for different scenarios (see [7] and the references therein) in the absence of the EH receiver.

\section{SYSTEM MODEL}

We consider a system shown in Fig. 1, which consists of a multi-antenna two-hop relaying system with a source, a relay, and a destination (also named as an information decoding (ID) receiver), and an EH receiver. The direct link between the source and ID receiver is not considered, since we assume that the effects of path attenuation and shadowing are more severe on the direct link than compared to the link via relay. Since, the relay operates in half-duplex mode, signal transmission in source-relay (S-R) and relay-destination 
(R-D) links takes place in two phases. In the first phase, the source encodes the input signal using OSTBC, precodes the encoded signal and transmits the resulting signal to the relay. The relay receives the source signal using the MRC technique and the resulting signal is normalized before applying the OSTBC. The relay finally precodes the latter signals and transmits the resulting signals in the second phase to the destination which employs MRC. The EH receiver receives signals from both the source and relay. The $n_{\mathrm{r}} \times T$ matrix of received signal samples at the relay can be given by $\mathbf{Y}_{\mathrm{r}}=\mathbf{H}_{1, \mathrm{I}} \mathbf{F}_{1} \mathbf{C}(\mathbf{s})+\mathbf{N}_{\mathrm{r}}$ where $\mathbf{s}=\left[s_{1}, \cdots, s_{K}\right]^{T}$ is $K \times 1$ information-bearing symbol vector, $\mathbf{C}(\mathbf{s})$ is the $n_{\mathrm{s}} \times T$ OSTBC matrix formed from $\mathbf{s}, T$ is the number of time periods used for transmitting $\mathbf{s}, \mathbf{H}_{1, \mathrm{I}} \in \mathcal{C}^{n_{\mathrm{r}} \times n_{\mathrm{s}}}$ is the S-R MIMO channel, $\mathbf{F}_{1} \in \mathcal{C}^{n_{\mathrm{s}} \times n_{\mathrm{s}}}$ is the source precoder, and $\mathbf{N}_{\mathrm{r}} \in$ $\mathcal{C}^{n_{\mathrm{r}} \times T}$ is the matrix of zero-mean circularly symmetric complex Gaussian (ZMCSCG) elements with variance $\tilde{\sigma}_{1}^{2}$. It is assumed that $\left\{s_{k}\right\}_{k=1}^{K}$ are chosen from signal constellations with $\mathrm{E}\left\{\left|s_{k}\right|^{2}\right\}=1$. Due to the orthogonality of OSTBC, $\mathbf{C}(\mathbf{s})$ fulfills the property $\mathbf{C}(\mathbf{s}) \mathbf{C}^{H}(\mathbf{s})=a\|\mathbf{s}\|^{2} \mathbf{I}_{n_{\mathrm{s}}}$, where the constant $a$ (e.g, $a=1$ for the Alamouti code [6]) depends on the chosen OSTBC matrix. The source power and the energy received by the $\mathrm{EH}$ receiver during the first phase of the two-hop transmission can be, respectively, given by

$$
P_{\mathrm{S}}=a K \operatorname{tr}\left(\mathbf{F}_{1} \mathbf{F}_{1}^{H}\right), P_{\mathrm{e}, 1}=a K \operatorname{tr}\left(\mathbf{H}_{1, \mathrm{E}} \mathbf{F}_{1} \mathbf{F}_{1}^{H} \mathbf{H}_{1, \mathrm{E}}^{H}\right)
$$

where $\mathbf{H}_{1, \mathrm{E}} \in \mathcal{C}^{n_{\mathrm{e}} \times n_{\mathrm{s}}}$ is the MIMO channel between the source and EH receiver. Due to the application of OSTBC at the source and the MRC scheme at the relay during the first phase of signal transmission, the S-R MIMO channel is decoupled into $K$ parallel SISO channels. Thus, the signal received by the relay on the $k$ th S-R SISO channel is given by

$$
y_{k}^{\mathrm{R}}=\left\|\mathbf{H}_{1, \mathrm{I}} \mathbf{F}_{1}\right\| s_{k}+n_{1, k}, k \in\{1, \cdots, K\}
$$

where $n_{1, k} \sim \mathcal{N}_{C}\left(0, \sigma_{1}^{2}\right)$ is the additive Gaussian noise at the relay for the $k$ th S-R SISO channel and $\sigma_{1}^{2}=\tilde{\sigma}_{1}^{2} / a$. The relay normalizes $\left\{y_{k}^{\mathrm{R}}\right\}_{k=1}^{K}$ yielding

$$
\tilde{y}_{k}^{\mathrm{R}}=\frac{y_{k}^{\mathrm{R}}}{\sqrt{\mathrm{E}\left\{\left|y_{k}^{\mathrm{R}}\right|^{2}\right\}}}=\frac{\left\|\mathbf{H}_{1, \mathrm{I}} \mathbf{F}_{1}\right\| s_{k}+n_{1, k}}{\sqrt{\left\|\mathbf{H}_{1, \mathrm{I}} \mathbf{F}_{1}\right\|^{2}+\sigma_{1}^{2}}} .
$$

The relay then employs OSTBC to encode $\left\{\tilde{y}_{k}^{\mathrm{R}}\right\}_{k=1}^{K}$ and precodes the resulting OSTBC encoded signal. The output of the relay is thus given by $\mathbf{Y}_{\text {ro }}=\mathbf{F}_{2} \mathbf{C}(\tilde{\mathbf{y}})$ where $\mathbf{F}_{2} \in \mathcal{C}^{n_{\mathrm{r}} \times n_{\mathrm{r}}}$ is the relay precoder, $\tilde{\mathbf{y}}=\left[\tilde{y}_{1}^{\mathrm{R}}, \cdots, \tilde{y}_{K}^{\mathrm{R}}\right]^{T}, \mathbf{C}(\tilde{\mathbf{y}}) \in \mathcal{C}^{n_{\mathrm{r}} \times T}$ is the OSTBC obtained after encoding $\tilde{\mathbf{y}}$ and satisfies the relation $\mathbf{C}(\tilde{\mathbf{y}}) \mathbf{C}^{H}(\tilde{\mathbf{y}})=a\|\tilde{\mathbf{y}}\|^{2} \mathbf{I}_{n_{\mathrm{r}}}$. The transmit power of the relay and the energy received by the EH receiver during the second time-phase can be thus, respectively, given by

$$
P_{\mathrm{r}}=a K \operatorname{tr}\left(\mathbf{F}_{2} \mathbf{F}_{2}^{H}\right), P_{\mathrm{e}, 2}=a K \operatorname{tr}\left(\mathbf{H}_{2, \mathrm{E}} \mathbf{F}_{2} \mathbf{F}_{2}^{H} \mathbf{H}_{2, \mathrm{E}}^{H}\right) .
$$

The $n_{\mathrm{d}} \times T$ matrix of signal samples received at the destina-

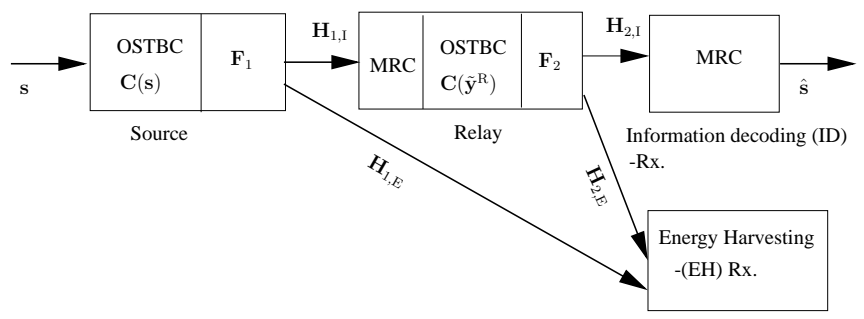

Fig. 1. Two-hop OSTBC based relay system with EH Rx.

tion during the second phase of transmission can be written as $\mathbf{Y}_{\mathrm{d}}=\mathbf{H}_{2, \mathrm{I}} \mathbf{F}_{2} \mathbf{C}(\tilde{\mathbf{y}})+\mathbf{N}_{\mathrm{d}}$, where $\mathbf{H}_{2, \mathrm{I}} \in \mathcal{C}^{n_{\mathrm{d}} \times n_{\mathrm{r}}}$ is the R-D MIMO channel and $\mathbf{N}_{\mathrm{d}} \in \mathcal{C}^{n_{\mathrm{d}} \times T}$ is the matrix of ZMCSCG elements with variance $\tilde{\sigma}_{2}^{2}$. The destination uses MRC to detect the source signals. Due to the application of OSTBC at the relay and MRC at the destination, the R-D MIMO channel also turns into $K$ parallel SISO channels. Thus, the signal received by the destination on the $k$ th R-D SISO channel can be expressed as

$$
y_{k, \mathrm{~d}}=\left\|\mathbf{H}_{2, \mathrm{I}} \mathbf{F}_{2}\right\| \tilde{y}_{k}^{\mathrm{R}}+n_{2, k}
$$

where $n_{2, k} \sim \mathcal{N}_{C}\left(0, \sigma_{2}^{2}\right)$ is the additive Gaussian noise at the destination for the $k$ th channel and $\sigma_{2}^{2}=\tilde{\sigma}_{2}^{2} / a$. With the help of (3), (5) can be written as

$$
y_{k, \mathrm{~d}}=\frac{\left\|\mathbf{H}_{2, \mathrm{I}} \mathbf{F}_{2}\right\|\left\|\mid \mathbf{H}_{1, \mathrm{I}} \mathbf{F}_{1}\right\| s_{k}+\mathbf{H}_{2, \mathrm{I}} \mathbf{F}_{2} n_{1, k}}{\sqrt{\left\|\mathbf{H}_{1, \mathrm{I}} \mathbf{F}_{1}\right\|^{2}+\sigma_{1}^{2}}}+n_{2, k} .
$$

The signal-to-noise ratio (SNR) at the destination can be expressed as

$$
\begin{aligned}
\gamma & =\frac{\left\|\mathbf{H}_{2, \mathrm{I}} \mathbf{F}_{2}\right\|^{2}\left\|\mathbf{H}_{1, \mathrm{I}} \mathbf{F}_{1}\right\|^{2}}{\left\|\mathbf{H}_{2, \mathrm{I}} \mathbf{F}_{2}\right\|^{2} \sigma_{1}^{2}+\left\|\mathbf{H}_{1, \mathrm{I}} \mathbf{F}_{1}\right\|^{2} \sigma_{2}^{2}+\sigma_{1}^{2} \sigma_{2}^{2}} \\
& =\frac{\gamma_{1} \gamma_{2}}{\gamma_{1}+\gamma_{2}+1}
\end{aligned}
$$

where $\gamma_{i}=\frac{\left\|\mathbf{H}_{i, \mathrm{I}} \mathbf{F}_{i}\right\|^{2}}{\sigma_{i}^{2}}$ for $i=1,2$.

\section{PROPOSED TRANSMISSION STRATEGIES}

First, we determine the optimal precoders $\mathbf{F}_{1}$ and $\mathbf{F}_{2}$ that maximize separately the received energy at the EH receiver and the information rate to the ID receiver. Consider the MIMO links from the source and relay to the $\mathrm{EH}$ receiver, when the ID receiver is not present. In this case, the objective is to design $\mathbf{F}_{1}$ and $\mathbf{F}_{2}$ to maximize the total power $P_{\mathrm{e}, 1}+P_{\mathrm{e}, 2}$ received at the $\mathrm{EH}$ receiver. This design problem can be formulated as

$$
\begin{aligned}
& \mathcal{P}_{1}: \max _{\mathbf{F}_{1}, \mathbf{F}_{2}} \operatorname{tr}\left(\mathbf{H}_{1, \mathrm{E}} \mathbf{F}_{1} \mathbf{F}_{1}^{H} \mathbf{H}_{1, \mathrm{E}}^{H}\right)+\operatorname{tr}\left(\mathbf{H}_{2, \mathrm{E}} \mathbf{F}_{2} \mathbf{F}_{2}^{H} \mathbf{H}_{2, \mathrm{E}}^{H}\right) \\
& \text { s.t. } \operatorname{tr}\left(\mathbf{F}_{1} \mathbf{F}_{1}^{H}\right)+\operatorname{tr}\left(\mathbf{F}_{2} \mathbf{F}_{2}^{H}\right) \leq P_{\mathrm{T}}
\end{aligned}
$$

where the constant $a K$ is omitted from the objective function, and $P_{\mathrm{T}}$ is given by $P_{\mathrm{T}}=\frac{\tilde{P}_{\mathrm{T}}}{a K}$, where $\tilde{P}_{\mathrm{T}}$ is the total power (source and relay). Let the eigen-decomposition (ED) 
of $\mathbf{H}_{i, \mathrm{E}}^{H} \mathbf{H}_{i, \mathrm{E}}$ be given by $\mathbf{H}_{i, \mathrm{E}}^{H} \mathbf{H}_{i, \mathrm{E}}=\mathbf{U}_{\mathbf{H}_{i, \mathrm{E}}} \boldsymbol{\Lambda}_{\mathbf{H}_{i, \mathrm{E}}} \mathbf{U}_{\mathbf{H}_{i, \mathrm{E}}}^{H}$ with eigenvalues $\lambda_{k}^{\mathbf{H}_{i, \mathrm{E}}}\left(k=1, \cdot \cdot r_{i} \triangleq \operatorname{rank}\left(\mathbf{H}_{i, \mathrm{E}}\right)\right)$, in the nondecreasing order, where $i=1,2$. Let $\left\{\mathbf{u}_{i, \mathrm{E}}\right\}_{i=1}^{2}$ be the column vectors of $\left\{\mathbf{U}_{\mathbf{H}_{i, \mathrm{E}}}\right\}_{i=1}^{2}$ corresponding to $\left\{\lambda_{1}^{\mathbf{H}_{i, \mathrm{E}}}\right\}_{i=1}^{2}$. Then, we have the following proposition.

Proposition 1: The optimal solutions to $\mathcal{P}_{1}$ are given by

$$
\begin{aligned}
& \tilde{\mathbf{F}}_{1}=\left[\sqrt{P_{\mathrm{T}}} \mathbf{u}_{1, \mathrm{E}}, \mathbf{0}, \cdots, \mathbf{0}\right], \tilde{\mathbf{F}}_{2}=\mathbf{0}, \text { if } \lambda_{1}^{\mathbf{H}_{1, \mathrm{E}}} \geq \lambda_{1}^{\mathbf{H}_{2, \mathrm{E}}} \\
& \tilde{\mathbf{F}}_{1}=\mathbf{0}, \tilde{\mathbf{F}}_{2}=\left[\sqrt{P_{\mathrm{T}}} \mathbf{u}_{2, \mathrm{E}}, \mathbf{0}, \cdots, \mathbf{0}\right], \text { if } \lambda_{1}^{\mathbf{H}_{1, \mathrm{E}}}<\lambda_{1}^{\mathbf{H}_{2, \mathrm{E}}} .(9)
\end{aligned}
$$

Proof: The proof is skipped due to space constraints.

Next, consider the two-hop MIMO relay link from the source to the ID receiver without the presence of the $\mathrm{EH}$ receiver. The optimal $\mathbf{F}_{1}$ and $\mathbf{F}_{2}$ that maximize the information rate over the two-hop MIMO channel can be obtained by solving the following problem

$$
\begin{aligned}
& \mathcal{P}_{2}: R_{\max } \triangleq \max _{\mathbf{F}_{1}, \mathbf{F}_{2}, \gamma_{1}, \gamma_{2}} \frac{1}{2} \ln _{2}\left(1+\frac{\gamma_{1} \gamma_{2}}{\gamma_{1}+\gamma_{2}+1}\right) \\
& \text { s.t } \gamma_{1}=\frac{\left\|\mathbf{H}_{1, \mathrm{I}} \mathbf{F}_{1}\right\|^{2}}{\sigma_{1}^{2}}, \gamma_{2}=\frac{\left\|\mathbf{H}_{2, \mathrm{I}} \mathbf{F}_{2}\right\|^{2}}{\sigma_{2}^{2}}, \\
& \operatorname{tr}\left(\mathbf{F}_{1} \mathbf{F}_{1}^{H}\right)+\operatorname{tr}\left(\mathbf{F}_{2} \mathbf{F}_{2}^{H}\right) \leq P_{\mathrm{T}} .
\end{aligned}
$$

Let the ED of $\mathbf{H}_{j, \mathrm{I}}^{H} \mathbf{H}_{j, \mathrm{I}}$ be given by $\mathbf{H}_{j, \mathrm{I}}^{H} \mathbf{H}_{j, \mathrm{I}}=\mathbf{U}_{\mathbf{H}_{j, \mathrm{I}}} \boldsymbol{\Lambda}_{\mathbf{H}_{j, \mathrm{I}}} \mathbf{U}_{\mathbf{H}_{j, \mathrm{I}}}^{H}$ with the eigenvalues $\lambda_{k}^{\mathbf{H}_{j, \mathrm{I}}}\left(k=1, \cdot \cdot r_{j} \triangleq \operatorname{rank}\left(\mathbf{H}_{j, \mathrm{I}}\right)\right)$ in the non-decreasing order, where $j=1,2$. Let $\left\{\mathbf{u}_{j, \mathrm{I}}\right\}_{j=1}^{2}$ be the column vectors of $\left\{\mathbf{U}_{\mathbf{H}_{j, \mathrm{I}}}\right\}_{j=1}^{2}$ corresponding to $\left\{\lambda_{1}^{\mathbf{H}_{j, \mathrm{I}}}\right\}_{j=1}^{2}$. It can be proved (the proof is omitted for brevity) that the optimal choices for $\mathbf{F}_{1}$ and $\mathbf{F}_{2}$ in $\mathcal{P}_{2}$ are

$$
\overline{\mathbf{F}}_{1}=\left[\sqrt{a_{1}} \mathbf{u}_{1, \mathrm{I}}, \mathbf{0}, \cdots, \mathbf{0}\right], \overline{\mathbf{F}}_{2}=\left[\sqrt{a_{2}} \mathbf{u}_{2, \mathrm{I}}, \mathbf{0}, \cdots, \mathbf{0}\right],
$$

where $a_{1} \geq 0, a_{2} \geq 0$ need to be optimized for $\mathcal{P}_{2}$. This leads to the following scalar-valued optimization problem for $\mathcal{P}_{2}$.

$$
\begin{aligned}
& \overline{\mathcal{P}}_{2}: R_{\max } \triangleq \max _{a_{1}, a_{2}} \frac{1}{2} \ln 2\left(1+\frac{a_{1} a_{2} \tilde{\lambda}_{1}^{\mathbf{H}_{1, \mathrm{I}}} \tilde{\lambda}_{1}^{\mathbf{H}_{2, \mathrm{I}}}}{a_{1} \tilde{\lambda}_{1}^{\mathbf{H}_{1, \mathrm{I}}}+a_{2} \tilde{\lambda}_{1}^{\mathbf{H}_{2, \mathrm{I}}}+1}\right) \\
& \text { s.t } a_{1}+a_{2} \leq P_{\mathrm{T}}
\end{aligned}
$$

where $\tilde{\lambda}_{1}^{\mathbf{H}_{1, \mathrm{I}}}=\frac{\lambda_{1}^{\mathbf{H}_{1, \mathrm{I}}}}{\sigma_{1}^{2}}$ and $\tilde{\lambda}_{1}^{\mathbf{H}_{2, \mathrm{I}}}=\frac{\lambda_{1}^{\mathbf{H}_{2, \mathrm{I}}}}{\sigma_{2}^{2}}$. It is easy to verify that the inequality $a_{1}+a_{2} \leq P_{\mathrm{T}}$ satisfies with equality at the optimality of $\overline{\mathcal{P}}_{2}$. Thus, substituting $a_{1}=P_{\mathrm{T}}-a_{2}$ into (12) and solving the first-order partial derivative of the objective function of $\overline{\mathcal{P}}_{2}$ w.r.t. $a_{2}$, we obtain

$$
\begin{aligned}
& \left(\tilde{\lambda}_{1}^{\mathbf{H}_{1, \mathrm{I}}}-\tilde{\lambda}_{1}^{\mathbf{H}_{2, \mathrm{I}}}\right) a_{2}^{2}-2\left(1+\tilde{\lambda}_{1}^{\mathbf{H}_{1, \mathrm{I}}} P_{\mathrm{T}}\right) a_{2}+ \\
& P_{\mathrm{T}}\left(1+\tilde{\lambda}_{1}^{\mathbf{H}_{1, \mathrm{I}}} P_{\mathrm{T}}\right)=0,
\end{aligned}
$$

which yields the following solution

$$
a_{2}=\frac{-u_{1} \pm \sqrt{u_{1}\left(1+\tilde{\lambda}_{1}^{\mathbf{H}_{2, \mathrm{I}}} P_{\mathrm{T}}\right)}}{\tilde{\lambda}_{1}^{\mathbf{H}_{1, \mathrm{I}}}-\tilde{\lambda}_{1}^{\mathbf{H}_{2, \mathrm{I}}}}
$$

where $u_{1}=1+\tilde{\lambda}_{1}^{\mathbf{H}_{1, \mathrm{I}}} P_{\mathrm{T}}$ and " - " is taken to guarantee that $0 \leq a_{2} \leq P_{\mathrm{T}}$. Then, $a_{1}$ is obtained from $a_{1}=P_{\mathrm{T}}-a_{2}$. Thus, the optimal solutions for $\mathcal{P}_{2}$ are derived.

We now consider the case where both the EH and ID receivers are present. To this end, our objective is to find the optimal transmission strategy for simultaneous wireless power and information transfer. For this purpose, we use the rateenergy (R-E) region which characterizes all the achievable rate and energy pairs for a given total power constraint of source and relays. We define the R-E region as

$$
\begin{aligned}
& \mathcal{C}_{R-E}\left(P_{\mathrm{T}}\right) \triangleq\left\{(R, P): R \leq \frac{1}{2} \ln _{2}\left(1+\frac{\gamma_{1} \gamma_{2}}{\gamma_{1}+\gamma_{2}+1}\right),\right. \\
& \gamma_{1}=\frac{\left\|\mathbf{H}_{1, \mathrm{I}} \mathbf{F}_{1}\right\|^{2}}{\sigma_{1}^{2}}, \gamma_{2}=\frac{\left\|\mathbf{H}_{2, \mathrm{I}} \mathbf{F}_{2}\right\|^{2}}{\sigma_{2}^{2}}, \\
& \left.P \leq\left\|\mathbf{H}_{1, \mathrm{E}} \mathbf{F}_{1}\right\|^{2}+\left\|\mathbf{H}_{2, \mathrm{E}} \mathbf{F}_{2}\right\|^{2},\left\|\mathbf{F}_{1}\right\|^{2}+\left\|\mathbf{F}_{2}\right\|^{2} \leq P_{\mathrm{T}}\right\}(15)
\end{aligned}
$$

Let $\left(R_{\mathrm{EH}}, P_{\max }\right)$ and $\left(R_{\max }, P_{\mathrm{ID}}\right)$ be the boundary points of this R-E region corresponding to the maximal power and information transfers, respectively. The source and relay precoders for the former boundary point are given by (9) which yield maximum power transfer of $P_{\max }=\left\|\mathbf{H}_{1, \mathrm{E}} \tilde{\mathbf{F}}_{1}\right\|^{2}+$ $\left\|\mathbf{H}_{2, \mathrm{E}} \tilde{\mathbf{F}}_{2}\right\|^{2}$ to the $\mathrm{EH}$ receiver and the information transfer of $R_{\mathrm{EH}}=0$ to the ID receiver. Note that no transfer of information to the ID receiver is obvious in this case, since the solution (9) means that either the source or the relay remains turned off. On the other hand, the source and relay precoders for the latter boundary point are given by (11) together with (14). With these precoders (i.e., $\overline{\mathbf{F}}_{1}$ and $\overline{\mathbf{F}}_{2}$ ), the information rate of $R_{\max }$ is achieved whereas the power transferred to the EH receiver becomes $P_{\mathrm{ID}}=\left\|\mathbf{H}_{1, \mathrm{E}} \overline{\mathbf{F}}_{1}\right\|^{2}+\left\|\mathbf{H}_{2, \mathrm{E}} \overline{\mathbf{F}}_{2}\right\|^{2}$. It can be easily seen that for $\bar{P} \leq P_{\mathrm{ID}}$, where $\bar{P} \geq 0$, the maximum rate $R_{\max }$ is achievable with the same $\overline{\mathbf{F}}_{1}$ and $\overline{\mathbf{F}}_{2}$ that achieve the R-E pair $\left(R_{\max }, P_{\mathrm{ID}}\right)$. The remaining boundary of R-E region that needs to be characterized is over the interval $P_{\text {ID }}<\bar{P}<P_{\max }$. For this purpose, we consider the following optimization problem with $\mathbf{W}_{1} \triangleq \mathbf{F}_{1} \mathbf{F}_{1}^{H} \succeq 0$ and $\mathbf{W}_{2} \triangleq \mathbf{F}_{2} \mathbf{F}_{2}^{H} \succeq 0$ :

$$
\begin{aligned}
& \mathcal{P}_{3}: \max _{\mathbf{W}_{1}, \mathbf{W}_{2}, \gamma_{1}, \gamma_{2}} \frac{1}{2} \ln _{2}\left(1+\frac{\gamma_{1} \gamma_{2}}{\gamma_{1}+\gamma_{2}+1}\right) \text { s.t } \\
& \operatorname{tr}\left(\mathbf{H}_{1, \mathrm{I}}^{H} \mathbf{H}_{1, \mathrm{I}} \mathbf{W}_{1}\right) \geq \gamma_{1} \sigma_{1}^{2}, \operatorname{tr}\left(\mathbf{H}_{2, \mathrm{I}}^{H} \mathbf{H}_{2, \mathrm{I}} \mathbf{W}_{2}\right) \geq \gamma_{2} \sigma_{2}^{2}(16 \mathrm{~b}) \\
& \operatorname{tr}\left(\mathbf{H}_{1, \mathrm{E}}^{H} \mathbf{H}_{1, \mathrm{E}} \mathbf{W}_{1}\right)+\operatorname{tr}\left(\mathbf{H}_{2, \mathrm{E}}^{H} \mathbf{H}_{2, \mathrm{E}} \mathbf{W}_{2}\right) \geq \bar{P} \\
& \operatorname{tr}\left(\mathbf{W}_{1}+\mathbf{W}_{2}\right) \leq P_{\mathrm{T}}, \mathbf{W}_{1} \succeq 0, \mathbf{W}_{2} \succeq 0 .
\end{aligned}
$$

Let $f\left(\gamma_{1}, \gamma_{2}\right)$ be defined as $f\left(\gamma_{1}, \gamma_{2}\right) \triangleq \frac{\gamma_{1} \gamma_{2}}{\gamma_{1}+\gamma_{2}+1}$. Then, we have the following Lemma for $f\left(\gamma_{1}, \gamma_{2}\right)$.

Lemma 1: $f\left(\gamma_{1}, \gamma_{2}\right)$ is a concave function.

Proof: This Lemma can be proved by showing that the Hessian matrix of $f\left(\gamma_{1}, \gamma_{2}\right)$ is negative definite. Due to limited space, the derivations have been omitted.

Since $f\left(\gamma_{1}, \gamma_{2}\right)$ is concave, $\ln _{2}\left(1+f\left(\gamma_{1}, \gamma_{2}\right)\right)$ is also concave [8]. Thus, the optimization problem $\mathcal{P}_{3}$ is convex since all of its constraints are linear w.r.t. $\mathbf{W}_{1}$ and $\mathbf{W}_{2}$ which in 
turn are constrained to be positive semidefinite. However, it is not easy to reformulate the convex optimization problem (16) into a suitable form so that standard convex optimization toolboxes [9] can be used. To circumvent this issue, we make the assumption that $\gamma_{1}+\gamma_{2}>>1$, which is accurate for moderate and high SNR values at the relay and destination. Thus, we approximate $f\left(\gamma_{1}, \gamma_{2}\right)$ by $\tilde{f}\left(\gamma_{1}, \gamma_{2}\right)$ where $\tilde{f}\left(\gamma_{1}, \gamma_{2}\right)=\frac{\gamma_{1} \gamma_{2}}{\gamma_{1}+\gamma_{2}}$. The resulting problem in (16) can be shown to have a semidefinite programming (SDP) formulation. For this purpose, we rewrite $\tilde{f}\left(\gamma_{1}, \gamma_{2}\right)$ as

$$
\tilde{f}\left(\gamma_{1}, \gamma_{2}\right)=\frac{1}{2}\left(\gamma_{1}+\gamma_{2}-\gamma_{1} \tilde{\gamma}^{-1} \gamma_{1}-\gamma_{2} \tilde{\gamma}^{-1} \gamma_{2}\right)
$$

where $\tilde{\gamma}=\gamma_{1}+\gamma_{2}$. Introducing a slack variable $\tau \geq 0$, we can re-express (16) as

$$
\begin{aligned}
& \overline{\mathcal{P}}_{3} \underset{\left\{\tau, \gamma_{i}, \mathbf{W}_{i}\right\}_{i=1}^{2}}{\max } \frac{1}{2} \ln _{2}(1+\tau) \text { s.t. }(16 b),(16 c),(16 d), \\
& 2 \tau \leq\left(\gamma_{1}+\gamma_{2}-\gamma_{1} \tilde{\gamma}^{-1} \gamma_{1}-\gamma_{2} \tilde{\gamma}^{-1} \gamma_{2}\right) .
\end{aligned}
$$

Applying Schur-complement theorem [8] twice in (18b), we can express it as

$$
\left[\begin{array}{cccc}
\gamma_{1}+\gamma_{2} & 0 & 0 & 0 \\
0 & \tilde{\gamma} & 0 & \gamma_{2} \\
0 & 0 & \gamma_{1}+\gamma_{2} & \gamma_{1} \\
0 & \gamma_{2} & \gamma_{1} & \gamma_{1}+\gamma_{2}-\tau
\end{array}\right] \succeq 0
$$

Finally, the following SDP formulation is obtained

$$
\overline{\mathcal{P}}_{3}: \max _{\left\{\tau, \gamma_{i}, \mathbf{W}_{i}\right\}_{i=1}^{2}} \frac{1}{2} \ln _{2}(1+\tau) \text { s.t. }(16 b),(16 c),(16 d),(19) \text {. }
$$

By solving the above problem $\overline{\mathcal{P}}_{3}$ for $P_{\mathrm{ID}}<\bar{P}<P_{\max }$, we obtain the optimal rate solutions that form the boundary of the $\mathrm{R}-\mathrm{E}$ region over the interval $R_{\mathrm{EH}}=0<R<R_{\max }$. Note that, in our case, the optimal $\left\{\mathbf{F}_{i}\right\}_{i=1}^{2}$ can be recovered from the optimal $\left\{\mathbf{W}_{i}\right\}_{i=1}^{2}$ without any loss of optimality.

\section{NUMERICAL RESULTS AND DISCUSSIONS}

We plot the boundary of the R-E region using numerical results. The results are obtained by averaging over 200 independent realizations of $\left\{\mathbf{H}_{i, \mathbf{I}}\right\}_{i=1}^{2}$ and $\left\{\mathbf{H}_{i, \mathbf{E}}\right\}_{i=1}^{2}$ whose elements are ZMCSCG random variables with unit variance. We take $\sigma_{1}^{2}=\sigma_{2}^{2}=\sigma^{2}$, and $n_{\mathrm{s}}=n_{\mathrm{r}}=2$, i.e., both the source and relay use Alamouti code [6]. Figure 2 plots the tradeoff between the maximum energy harvested by the $\mathrm{EH}$ receiver and the maximum information rate transferred to the ID receiver for different values of average $\operatorname{SNR}\left(\gamma_{\mathrm{av}} \triangleq \tilde{P}_{\mathrm{T}} / \sigma^{2}\right)$, $n_{\mathrm{d}}$, and $n_{\mathrm{e}}$. As can be seen from this figure, for the given $n_{\mathrm{d}}$ and $n_{\mathrm{e}}$, the information transfer increases as $\gamma_{\mathrm{av}}$ increases whereas the power transfer remains almost the same. The latter also remains unchanged when $n_{\mathrm{d}}$ increases for the given $\gamma_{\mathrm{av}}$ and $n_{\mathrm{e}}$. However, the power transfer to the EH receiver improves when $n_{\mathrm{e}}$ increases for the given $\gamma_{\mathrm{av}}$ and $n_{\mathrm{d}}$. Hence, based on the results of Figure 2, it can be concluded that the larger values of $\gamma_{\mathrm{av}}$ and $n_{\mathrm{d}}$ are desired for having better information rate transfer whereas the larger value of $n_{\mathrm{e}}$ is required for improving the energy transfer to the $\mathrm{EH}$ receiver.

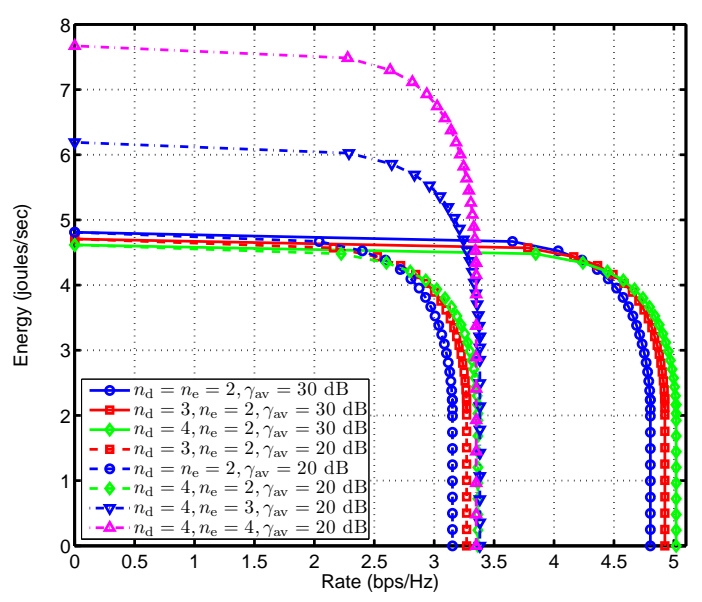

Fig. 2. R-E region tradeoff for the considered system.

\section{CONCLUSIONS}

We have provided the performance limits of OSTBC based MIMO cooperative relay system that allows low-powered wireless devices in its vicinity to harvest energy. The tradeoffs in information rate and energy transfer were characterized by the boundary of the R-E region which is obtained by solving joint source and relay precoder optimization problems.

\section{REFERENCES}

[1] R. Want, "Enabling ubiquitous sensing with RFID," IEEE Computer, vol. 37, pp. 84-86, Apr. 2004.

[2] F. Zhang et al., "Wireless energy transfer platform for medical sensors and implantable devices," in Proc. IEEE EMBS 31 st Annual Int. Conf., pp. 1045-1048, Sept. 2009.

[3] R. Zhang and C. K. Ho, "MIMO broadcasting for simultaneous wireless information and power transfer," http://arxiv.org/abs/1105.4999, May 2011.

[4] L. R. Varshney, "Transporting information and energy simultaneously," in Proc. IEEE Int. Symp. Inf. Theory (ISIT), pp. 16121616, July 2008.

[5] P. Grover and A. Sahai, "Shannon meets Tesla: wireless information and power transfer," in Proc. IEEE Int. Symp. Inf. Theory (ISIT), pp. 2363-2367, June 2010.

[6] V. Tarokh, H. Jafharkani, and A. R. Calderbank, "Space-time block codes from orthogonal designs," IEEE Trans. Info. Theo., vol. 45, no. 5, pp. 1456-1467, July 1999.

[7] B. K. Chalise and L. Vandendorpe, "MIMO relay design for multipoint-to-multipoint communications with imperfect channel state information," IEEE Trans. Sig. Proc., vol. 57, no. 7, pp. 2785-2796, July 2009.

[8] S. Boyd and L. Vandenberghe, Convex Optimization, Cambridge University Press, 2004.

[9] M. Grant and S. Boyd, CVX: Matlab Software for Disciplined Convex Programming, http://stanford.edu//boyd/cvx. 